\title{
Compiling finite linear CSP into SAT
}

\author{
Naoyuki Tamura • Akiko Taga • \\ Satoshi Kitagawa Mutsunori Banbara
}

Published online: 24 December 2008

(C) The Author(s) 2008. This article is published with open access at Springerlink.com

\begin{abstract}
In this paper, we propose a new method to encode Constraint Satisfaction Problems (CSP) and Constraint Optimization Problems (COP) with integer linear constraints into Boolean Satisfiability Testing Problems (SAT). The encoding method (named order encoding) is basically the same as the one used to encode JobShop Scheduling Problems by Crawford and Baker. Comparison $x \leq a$ is encoded by a different Boolean variable for each integer variable $x$ and integer value $a$. To evaluate the effectiveness of this approach, we applied the method to the OpenShop Scheduling Problems (OSS). All 192 instances in three OSS benchmark sets are examined, and our program found and proved the optimal results for all instances including three previously undecided problems.
\end{abstract}

Keywords Constraint satisfaction problems • SAT encoding • Open-shop scheduling problems

\section{Introduction}

Recent advances in SAT solver technologies [6, 16-18, 20] have enabled solving a problem by encoding it as a SAT problem, and then using an efficient SAT solver to find a solution, such as for model checking, planning, and scheduling [4, 7, 10, 11, $14,19,21]$.

In this paper, we propose a new method to encode Constraint Satisfaction Problems (CSP) and Constraint Optimization Problems (COP) with integer linear

N. Tamura $(\bowtie) \cdot$ M. Banbara

Information Science and Technology Center, Kobe University, Kobe, Japan e-mail: tamura@kobe-u.ac.jp

\footnotetext{
A. Taga $\cdot$ S. Kitagawa

Graduate School of Science and Technology, Kobe University, Kobe, Japan

空 Springer
} 
constraints into Boolean Satisfiability Testing Problems (SAT) of CNF (product-ofsums) formulas [23].

As Hoos discussed in [10], basically two encoding methods are known: "sparse encoding" and "compact encoding". Sparse encoding [5] encodes each assignment of a value to an integer variable by a different Boolean variable, that is, Boolean variable representing $x=a$ is used for each integer variable $x$ and integer value $a$. The direct encoding [24] and the support encoding [8] are based on the sparse encoding. The compact encoding $[7,12]$ assigns a Boolean variable for each bit of each integer variable.

The encoding method used in this paper (named order encoding) is different from these. The method is basically the same as the one used to encode Job-Shop

Scheduling Problems by Crawford and Baker in [4] and studied by Soh, Inoue, and Nabeshima in $[11,19,21]$. It encodes a comparison $x \leq a$ by a different Boolean variable for each integer variable $x$ and integer value $a$.

The benefit of this encoding is the natural representation of the order relation on integers. Axiom clauses with two literals, such as $\{\neg(x \leq a), x \leq a+1\}$ for each integer $a$, represent the order relation for an integer variable $x$. Clauses, for example $\{x \leq a, \neg(y \leq a)\}$ for each integer $a$, can be used to represent the constraint among integer variables, i.e. $x \leq y$.

The original encoding method used in $[4,11,19,21]$ is only for Job-Shop Scheduling Problems. In this paper, we extend the method so that it can be applied to any finite linear CSPs and COPs.

To evaluate the effectiveness of this approach, we applied the method to the Graph Coloring Problems and the Open-Shop Scheduling Problems (OSS).

The proposed method gives the better performance compared with the direct encoding [24] and the support encoding [8] for the Graph Coloring Problems.

As for OSS problems, all 192 instances in three OSS benchmark sets proposed in $[3,9,22]$ are examined, and our program found and proved the optimal results for all instances including three previously undecided problems $[2,13,15]$.

\section{Finite linear CSP and SAT}

In this section, we define finite linear Constraint Satisfaction Problems (CSP) and Boolean Satisfiability Testing Problems (SAT) of CNF formulas.

$\mathbf{Z}$ is used to denote a set of integers and $\mathbf{B}$ is used to denote a set of Boolean constants ( $\top$ and $\perp$ are the only elements of $\mathbf{B}$ representing "true" and "false" respectively).

We also prepare two countably infinite sets of integer variables $\mathcal{V}$ and Boolean variables $\mathcal{B}$. Although only a finite number of variables are used in a specific CSP or SAT, countably infinite variables are prepared to introduce new variables during the translation. Symbols $x, y, z, x_{1}, y_{1}, z_{1}, \ldots$, are used to denote integer variables, and symbols $p, q, r, p_{1}, q_{1}, r_{1}, \ldots$, are used to denote Boolean variables.

Linear expressions over $V \subset \mathcal{V}$, denoted by $E(V)$, are algebraic expressions in the form of $\sum a_{i} x_{i}$ where $a_{i}$ 's are non-zero integers and $x_{i}$ 's are integer variables (elements of $V$ ). We also add the restriction that $x_{i}$ 's are mutually distinct.

Literals over $V \subset \mathcal{V}$ and $B \subset \mathcal{B}$, denoted by $L(V, B)$, consist of Boolean variables $\{p \mid p \in B\}$, negations of Boolean variables $\{\neg p \mid p \in B\}$, and comparisons $\{e \leq c \mid$ $e \in E(V), c \in \mathbf{Z}\}$. Please note that we restrict comparison literals to only appear 
positively and in the form of $\sum a_{i} x_{i} \leq c$ without loss of generality. For example, $\neg\left(a_{1} x_{1}+a_{2} x_{2} \leq c\right)$ can be represented with $-a_{1} x_{1}-a_{2} x_{2} \leq-c-1$, and $x \neq y$ (that is, $(x<y) \vee(x>y))$ can be represented with $(x-y \leq-1) \vee(-x+y \leq-1)$. Encoding of other expressions, such as max, abs, etc., will be explained in Section 3.5.

Clauses over $V \subset \mathcal{V}$ and $B \subset \mathcal{B}$, denoted by $C(V, B)$, are defined as usual where literals are chosen from $L(V, B)$, that is, a clause represents a disjunction of element literals. Integer variables occurring in a clause are treated as free variables, that is, a clause $\{x \leq 0\}$ does not mean $\forall x$. $(x \leq 0)$.

Definition 1 (Finite linear CSP) A (finite linear) CSP (Constraint Satisfaction Problem) is defined as a tuple $(V, \ell, u, B, S)$ where

(1) $V$ is a finite subset of integer variables $\mathcal{V}$,

(2) $\ell$ is a mapping from $V$ to $\mathbf{Z}$ representing the lower bound of the integer variable,

(3) $u$ is a mapping from $V$ to $\mathbf{Z}$ representing the upper bound of the integer variable,

(4) $B$ is a finite subset of Boolean variables $\mathcal{B}$, and

(5) $S$ is a finite set of clauses (that is, a finite subset of $C(V, B)$ ) representing the constraint to be satisfied.

In the rest of this paper, we simply call finite linear CSP as CSP.

We extend the functions $\ell$ and $u$ for any linear expressions $e \in E(V)$, e.g. $\ell(2 x-3 y)=-9$ and $u(2 x-3 y)=6$ when $\ell(x)=\ell(y)=0$ and $u(x)=u(y)=3$.

An assignment of a $\operatorname{CSP}(V, \ell, u, B, S)$ is a pair $(\alpha, \beta)$ where $\alpha$ is a mapping from $V$ to $\mathbf{Z}$ and $\beta$ is a mapping from $B$ to $\{\top, \perp\}$.

Definition 2 (Satisfiability) Let $(V, \ell, u, B, S)$ be a CSP. A clause $C \in C(V, B)$ is satisfiable by an assignment $(\alpha, \beta)$ if the assignment makes the clause $C$ be true and $\ell(x) \leq \alpha(x) \leq u(x)$ for all $x \in V$. We denote this satisfiability relation as follows.

$$
(\alpha, \beta) \models C
$$

A clause $C$ is satisfiable if $C$ is satisfiable by some assignment.

A set of clauses is satisfiable when all clauses in the set are satisfiable by the same assignment. A logical formula is satisfiable when its clausal form is satisfiable. The CSP is satisfiable if the set of clauses $S$ is satisfiable.

Finally, we define SAT as a special form of CSP.

Definition 3 (SAT) A SAT (Boolean Satisfiability Testing Problem) is a CSP without integer variables, that is, $(\emptyset, \emptyset, \emptyset, B, S)$.

\section{Encoding finite linear CSP to SAT}

\subsection{Converting comparisons to primitive comparisons}

In this section, we will explain a method to transform a comparison into primitive comparisons.

A primitive comparison is a comparison in the form of $x \leq c$ where $x$ is an integer variable and $c$ is an integer satisfying $\ell(x)-1 \leq c \leq u(x)$. In fact, it is possible to 
restrict the range of $c$ to $\ell(x) \leq c \leq u(x)-1$ since $x \leq \ell(x)-1$ is always false and $x \leq u(x)$ is always true. However, we use the wider range to simplify the discussion.

Let us consider a comparison of $x+y \leq 7$ when $\ell(x)=\ell(y)=2$ and $u(x)=$ $u(y)=6$. As shown in Fig. 1, the comparison can be equivalently expressed as $(x \leq$ $1 \vee y \leq 5) \wedge(x \leq 2 \vee y \leq 4) \wedge(x \leq 3 \vee y \leq 3) \wedge(x \leq 4 \vee y \leq 2) \wedge(x \leq 5 \vee y \leq 1)$ in which 10 black dotted points are contained as satisfiable assignments since $0 \leq x$, $y \leq 6$. Please note that conditions $(x \leq 1 \vee y \leq 5)$ and $(x \leq 5 \vee y \leq 1)$, which are equivalent to $y \leq 5$ and $x \leq 5$ respectively, are necessary to exclude cases of $x=2$, $y=6$ and $x=6, y=2$.

Now, we will show the following lemma before describing the conversion to primitive comparisons in general.

Lemma 1 Let $(V, \ell, u, B, S)$ be a $C S P$, then for any assignment $(\alpha, \beta)$ of the CSP, for any linear expressions $e, f \in E(V)$, and for any integer $c \geq \ell(e)+\ell(f)$, the following holds.

$$
\begin{aligned}
(\alpha, \beta) & \models e+f \leq c \\
\Longleftrightarrow \quad(\alpha, \beta) & \models \bigwedge_{a+b=c-1}(e \leq a \vee f \leq b)
\end{aligned}
$$

Parameters $a$ and $b$ range over $\mathbf{Z}$ satisfying $a+b=c-1, \ell(e)-1 \leq a \leq u(e)$, and $\ell(f)-1 \leq b \leq u(f)$. The conjunction represents $\top$ if there are no such $a$ and $b$.

Proof $(\Longrightarrow)$ From the hypotheses and the definition of satisfiability, we get $\alpha(e)+$ $\alpha(f) \leq c, \ell(e) \leq \alpha(e) \leq u(e)$, and $\ell(f) \leq \alpha(f) \leq u(f)$. Let $a$ and $b$ be any integers satisfying $a+b=c-1, \ell(e)-1 \leq a \leq u(e)$, and $\ell(f)-1 \leq b \leq u(f)$. If there are no such $a$ and $b$, the conclusion holds.

Fig. 1 Converting $x+y \leq 7$ to primitive comparisons

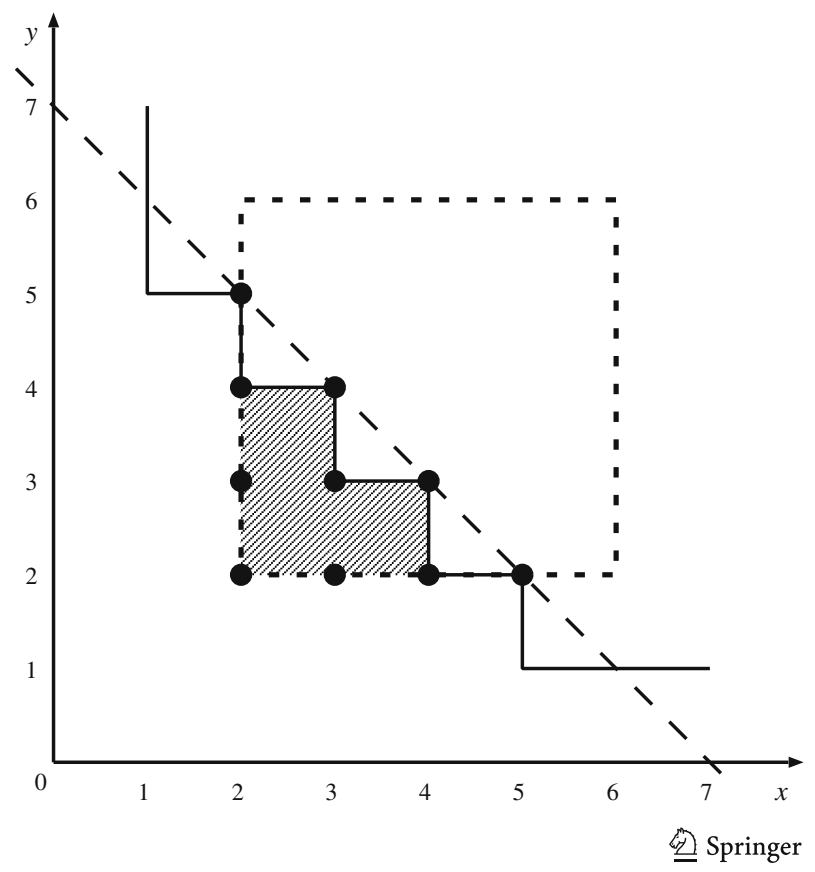


If $\alpha(e) \leq a, e \leq a$ in the conclusion is satisfied. Otherwise, $f \leq b$ in the conclusion is satisfied since $\alpha(f) \leq c-\alpha(e) \leq c-a-1=(a+b+1)-a-1=b$. Therefore, $e \leq a \vee f \leq b$ is satisfied for any $a$ and $b$.

( $\Longleftarrow$ ) From the hypotheses, $\alpha(e) \leq a \vee \alpha(f) \leq b$ is true for any $a$ and $b$ satisfying $a+b=c-1, \ell(e)-1 \leq a \leq u(e)$, and $\ell(f)-1 \leq b \leq u(f)$. From the definition of satisfiability, we also have $\ell(e) \leq \alpha(e) \leq u(e)$ and $\ell(f) \leq \alpha(f) \leq u(f)$. Now, we show the conclusion through a proof by contradiction. Assume that $\alpha(e)+\alpha(f)>c$ which is the negation of the conclusion.

When $\alpha(e) \geq c-\ell(f)+1$, we choose $a=c-\ell(f)$ and $b=\ell(f)-1$. It is easy to check the conditions $\ell(e)-1 \leq a \leq u(e)$ and $\ell(f)-1 \leq b \leq u(f)$ are satisfied, and $\alpha(e) \leq a \vee \alpha(f) \leq b$ becomes false for such $a$ and $b$, which contradicts the hypotheses.

When $\alpha(e)<c-\ell(f)+1$, we choose $a=\alpha(e)-1$ and $b=c-\alpha(e)$. It is easy to check the conditions $\ell(e)-1 \leq a \leq u(e)$ and $\ell(f)-1 \leq b \leq u(f)$ are satisfied, and $\alpha(e) \leq a \vee \alpha(f) \leq b$ becomes false for such $a$ and $b$, which contradicts the hypotheses.

The following proposition shows a general method to convert a (linear) comparison into primitive comparisons.

Proposition 1 Let $(V, \ell, u, B, S)$ be a CSP, then for any assignment $(\alpha, \beta)$ of the $C S P$, for any linear expression $\sum_{i=1}^{n} a_{i} x_{i} \in E(V)$, and for any integer $c \geq \ell\left(\sum_{i=1}^{n} a_{i} x_{i}\right)$ the following holds.

$$
\begin{gathered}
(\alpha, \beta) \models \sum_{i=1}^{n} a_{i} x_{i} \leq c \\
\Longleftrightarrow \quad(\alpha, \beta) \models \bigwedge_{\sum_{i=1}^{n} b_{i}=c-n+1} \bigvee_{i}\left(a_{i} x_{i} \leq b_{i}\right)^{\#}
\end{gathered}
$$

Parameters $b_{i}$ 's range over $\mathbf{Z}$ satisfying $\sum_{i=1}^{n} b_{i}=c-n+1$ and $\ell\left(a_{i} x_{i}\right)-1 \leq b_{i} \leq$ $u\left(a_{i} x_{i}\right)$ for all $i$. The translation ( $)^{\#}$ is defined as follows.

$$
(a x \leq b)^{\#} \equiv \begin{cases}x \leq\left\lfloor\frac{b}{a}\right\rfloor & (a>0) \\ \neg\left(x \leq\left\lceil\frac{b}{a}\right\rceil-1\right) & (a<0)\end{cases}
$$

Proof The satisfiability of $\sum a_{i} x_{i} \leq c$ is equivalent to the satisfiability of $\bigwedge \bigvee\left(a_{i} x_{i} \leq\right.$ $b_{i}$ ) from Lemma 1 , and the satisfiability of each $a_{i} x_{i} \leq b_{i}$ is equivalent to the satisfiability of $\left(a_{i} x_{i} \leq b_{i}\right)^{\#}$.

Therefore, any comparison literal $\sum a_{i} x_{i} \leq c$ in a CSP can be converted to a CNF (product-of-sums) formula of primitive comparisons (or Boolean constants) without changing its satisfiability. Please note that the comparison literal should occur positively in the CSP to perform this conversion.

Example 1 When $\ell(x)=\ell(y)=\ell(z)=0$ and $u(x)=u(y)=u(z)=3$, comparison $x+y<z-1$ is converted into $(x \leq-1 \vee y \leq-1 \vee \neg(z \leq 1)) \wedge(x \leq-1 \vee y \leq$ $0 \vee \neg(z \leq 2)) \wedge(x \leq-1 \vee y \leq 1 \vee \neg(z \leq 3)) \wedge(x \leq 0 \vee y \leq-1 \vee \neg(z \leq 2)) \wedge(x \leq$ $0 \vee y \leq 0 \vee \neg(z \leq 3)) \wedge(x \leq 1 \vee y \leq-1 \vee \neg(z \leq 3))$. 


\subsection{Encoding to SAT}

As shown in the previous subsection, any (finite linear) CSP can be converted into a CSP with only primitive comparisons.

Now, we eliminate each primitive comparison $x \leq c(x \in V, \ell(x)-1 \leq c \leq u(x))$ by replacing it with a newly introduced Boolean variable $p(x, c)$ which is chosen from $\mathcal{B}$. We denote a set of these new Boolean variables as follows.

$$
B^{\prime}=\{p(x, c) \mid x \in V, \ell(x)-1 \leq c \leq u(x)\}
$$

We also need to introduce the following axiom clauses $A(x)$ for each integer variable $x$ in order to represent the bound and the order relation.

$$
\begin{aligned}
A(x)= & \{\{\neg p(x, \ell(x)-1)\},\{p(x, u(x))\}\} \\
& \cup\{\{\neg p(x, c-1), p(x, c)\} \mid \ell(x) \leq c \leq u(x)\}
\end{aligned}
$$

As previously described, clauses of $\{\neg p(x, \ell(x)-1)\}$ and $\{p(x, u(x))\}$ are redundant. However, these will be removed in the early stage of SAT solving and will not affect much the performance of the solver.

Proposition 2 Let $(V, \ell, u, B, S)$ be a CSP with only primitive comparisons, let $S^{*}$ be a clausal form formula obtained from $S$ by replacing each primitive comparison $x \leq c$ with $p(x, c)$, and let $A=\bigcup_{x \in V} A(x)$. Then, the following holds.

$$
\begin{aligned}
&(V, \ell, u, B, S) \text { is satisfiable } \\
& \Longleftrightarrow \quad\left(\emptyset, \emptyset, \emptyset, B \cup B^{\prime}, S^{*} \cup A\right) \text { is satisfiable }
\end{aligned}
$$

Proof $(\Longrightarrow)$ Since $(V, \ell, u, B, S)$ is satisfiable, there is an assignment $(\alpha, \beta)$ which makes $S$ be true and $\ell(x) \leq \alpha(x) \leq u(x)$ for all $x \in V$. We extend the mapping $\beta$ to $\beta^{*}$ as follows.

$$
\beta^{*}(p)= \begin{cases}\beta(p) & (p \in B) \\ \alpha(x) \leq c & \left(p=p(x, c) \in B^{\prime}\right)\end{cases}
$$

Then an assignment $\left(\alpha, \beta^{*}\right)$ satisfies $S^{*} \cup A$.

$(\Longleftarrow)$ From the hypotheses, there is an assignment $(\varnothing, \beta)$ which makes $S^{*} \cup A$ be true. We define a mapping $\alpha$ as follows.

$$
\alpha(x)=\min \{c \mid \ell(x) \leq c \leq u(x), p(x, c)\}
$$

It is straightforward to check the assignment $(\alpha, \beta)$ satisfies $S$.

\subsection{Keeping clausal form}

When encoding a clause of CSP to SAT, the encoded formula is no more a clausal form in general.

Consider a case of encoding a clause $\{x-y \leq-1,-x+y \leq-1\}$ which means $x \neq$ $y$. Each of $x-y \leq-1$ and $-x+y \leq-1$ is encoded into a CNF formula of primitive comparisons. Therefore, when we expand the conjunctions to get a clausal form, the number of obtained clauses is the multiplication of two numbers of primitive comparisons. 
As it is well known, introduction of new Boolean variables is useful to reduce the size. Suppose $\left\{c_{1}, c_{2}, \ldots, c_{n}\right\}$ is a clause of original CSP where $c_{i}$ 's are comparison literals, and $\left\{C_{i 1}, C_{i 2}, \ldots, C_{i n_{i}}\right\}$ is an encoded CNF formula (in clausal form) of $c_{i}$ for each $i$.

We introduce new Boolean variables $p_{1}, p_{2}, \ldots, p_{n}$ chosen from $\mathcal{B}$, and replace the original clause with $\left\{p_{1}, p_{2}, \ldots, p_{n}\right\}$. We also introduce new clauses $\left\{\neg p_{i}\right\} \cup C_{i j}$ for each $1 \leq i \leq n$ and $1 \leq j \leq n_{i}$.

This conversion does not affect the satisfiability which can be shown from the following lemma.

Lemma 2 Let $(V, \ell, u, B, S)$ be a CSP, $\left\{L_{1}, L_{2}, \ldots, L_{n}\right\}$ be a clause of the CSP, and $p_{1}, p_{2}, \ldots, p_{n}$ be new Boolean variables. Then, the following holds.

$$
\begin{aligned}
& \left\{L_{1}, L_{2}, \ldots, L_{n}\right\} \text { is satisfiable } \\
\Longleftrightarrow \quad & \left\{\left\{p_{1}, p_{2}, \ldots, p_{n}\right\}\left\{\neg p_{1}, L_{1}\right\},\left\{\neg p_{2}, L_{2}\right\}, \ldots,\left\{\neg p_{n}, L_{n}\right\}\right\} \text { is satisfiable }
\end{aligned}
$$

Proof $(\Longrightarrow)$ From the hypotheses, there is an assignment $(\alpha, \beta)$ which satisfies some $L_{i}$. We extend the mapping $\beta$ so that $\beta\left(p_{i}\right)=\top$ and $\beta\left(p_{j}\right)=\perp(j \neq i)$. Then, the assignment satisfies converted clauses.

$(\Longleftarrow)$ From the hypotheses, there is an assignment $(\alpha, \beta)$ which satisfies some $p_{i}$. The assignment also satisfies $\left\{\neg p_{i}, L_{i}\right\}$, and therefore $L_{i}$. Hence the conclusion holds.

Example 2 Consider an example of encoding a clause $\{x-y \leq-1,-x+y \leq-1\}$ when $\ell(x)=\ell(y)=0$ and $u(x)=u(y)=2 . x-y \leq-1$ and $-x+y \leq-1$ are converted into $S_{1}=(p(x,-1) \vee \neg p(y, 0)) \wedge(p(x, 0) \vee \neg p(y, 1)) \wedge(p(x, 1) \vee \neg p(y, 2))$ and $S_{2}=(\neg p(x, 2) \vee p(y, 1)) \wedge(\neg p(x, 1) \vee p(y, 0)) \wedge(\neg p(x, 0) \vee p(y,-1))$ respectively. Expanding $S_{1} \vee S_{2}$ generates 9 clauses. However, by introducing new Boolean variables $p$ and $q$, we obtain the following seven clauses.

$$
\begin{array}{ccc} 
& \{p, q\} & \\
\{\neg p, p(x,-1), \neg p(y, 0)\} & \{\neg p, p(x, 0), \neg p(y, 1)\} & \{\neg p, p(x, 1), \neg p(y, 2)\} \\
\{\neg q, \neg p(x, 2), p(y, 1)\} & \{\neg q, \neg p(x, 1), p(y, 0)\} & \{\neg q, \neg p(x, 0), p(y,-1)\}
\end{array}
$$

\subsection{Size of the encoded SAT problem}

Usually the size of the encoded SAT problem becomes large.

Suppose the number of integer variables is $n$, and the size of integer variable domains is $d$, that is, $d=u(x)-\ell(x)+1$ for all $x \in V$. Then the size of newly introduced Boolean variables $B^{\prime}$ is $O(n d)$, the size of axiom clauses $A$ is also $O(n d)$, and the number of literals in each axiom clause is at most two.

Each comparison $\sum_{i=1}^{m} a_{i} x_{i} \leq c$ will be encoded into $O\left(d^{m-1}\right)$ clauses in general by Proposition 1.

However, it is possible to reduce the number of integer variables in each comparison to at most three. For example, $x_{1}+x_{2}+x_{3}+x_{4} \leq c$ can be replaced with $x+x_{3}+x_{4} \leq c$ by introducing a new integer variable $x$ and new constraints $x-x_{1}-$ $x_{2} \leq 0$ and $-x+x_{1}+x_{2} \leq 0$, that is, $x=x_{1}+x_{2}$.

Therefore, each comparison $\sum_{i=1}^{m} a_{i} x_{i} \leq c$ can be encoded by at most $O\left(m d^{2}\right)$ clauses even when $m \geq 4$, and the number of literals in each clause is at most four 
(three for integer variables and one for the case handling described in the previous subsection).

\subsection{Encoding expressions other than $\sum a_{i} x_{i} \leq b$}

Comparisons and expressions other than $\sum a_{i} x_{i} \leq b$ can also be encoded to SAT by using the conversion described in Fig. 2.

Comparisons and expressions (the first column) can be replaced with the replacement (the second column) with some extra condition (the third column) where $E \operatorname{div} c$ and $E \bmod c$ are integer quotient and remainder of $E$ divided by an integer constant $c$.

\subsection{Comparison with direct and support encodings}

This section compares the proposed encoding with other encodings, especially the direct encoding [24] and the support encoding [8], through the following CSP example.

$$
\begin{aligned}
x & \in\{2,3,4,5,6\} \\
y & \in\{2,3,4,5,6\} \\
x+y & \leq 7
\end{aligned}
$$

Direct encoding: The direct encoding [24] uses Boolean variables $p_{x i}$ meaning $x=i$ for each CSP variable $x$ and each integer constant $i(\ell(x) \leq i \leq u(x))$. Therefore, 10 Boolean variables are used for the above CSP example.

$$
\begin{array}{lllll}
p_{x 2} & p_{x 3} & p_{x 4} & p_{x 5} & p_{x 6} \\
p_{y 2} & p_{y 3} & p_{y 4} & p_{y 5} & p_{y 6}
\end{array}
$$

The following at-least-one and at-most-one clauses are used as axioms for each CSP variable $x$.

$$
\begin{aligned}
& p_{x \ell(x)} \vee \cdots \vee p_{x u(x)} \\
& \neg p_{x i} \vee \neg p_{x j} \quad(\ell(x) \leq i<j \leq u(x))
\end{aligned}
$$

\begin{tabular}{ccc}
\hline Expression & Replacement & Extra condition \\
\hline$E<F$ & $E+1 \leq F$ & \\
$E=F$ & $(E \leq F) \wedge(E \geq F)$ & \\
$E \neq F$ & $(E<F) \vee(E>F)$ & \\
$\max (E, F)$ & $x$ & $(x \geq E) \wedge(x \geq F) \wedge((x \leq E) \vee(x \leq F))$ \\
$\min (E, F)$ & $x$ & $(x \leq E) \wedge(x \leq F) \wedge((x \geq E) \vee(x \geq F))$ \\
$\operatorname{abs}(E)$ & $\max (E,-E)$ & $(E=c q+r) \wedge(0 \leq r) \wedge(r<c)$ \\
$E \operatorname{div} c$ & $q$ & $(E=c q+r) \wedge(0 \leq r) \wedge(r<c)$ \\
$E \bmod c$ & $r$ & \\
\hline
\end{tabular}

Fig. 2 Encoding expressions other than $\sum a_{i} x_{i} \leq b$ 
Therefore, the following 22 clauses are required for the example.

$$
\begin{gathered}
p_{x 2} \vee p_{x 3} \vee p_{x 4} \vee p_{x 5} \vee p_{x 6} \\
\neg p_{x 2} \vee \neg p_{x 3} \quad \neg p_{x 2} \vee \neg p_{x 4} \quad \neg p_{x 2} \vee \neg p_{x 5} \\
\neg p_{x 2} \vee \neg p_{x 6} \quad \neg p_{x 3} \vee \neg p_{x 4} \quad \neg p_{x 3} \vee \neg p_{x 5} \\
\neg p_{x 3} \vee \neg p_{x 6} \quad \neg p_{x 4} \vee \neg p_{x 5} \quad \neg p_{x 4} \vee \neg p_{x 6} \quad \neg p_{x 5} \vee \neg p_{x 6} \\
\text { (similar clauses for y) }
\end{gathered}
$$

Constraints are encoded into clauses representing conflict points. When $x_{1}=i_{1}$, $\ldots, x_{n}=i_{n}$ violates the constraint, the following clause is added.

$$
\neg p_{x_{1} i_{1}} \vee \cdots \vee \neg p_{x_{n} i_{n}}
$$

Therefore, 15 clauses are used to encode $x+y \leq 7$.

$$
\begin{array}{lllll}
\neg p_{x 2} \vee \neg p_{y 6} & \neg p_{x 3} \vee \neg p_{y 5} & \neg p_{x 3} \vee \neg p_{y 6} & \neg p_{x 4} \vee \neg p_{y 4} & \neg p_{x 4} \vee \neg p_{y 5} \\
\neg p_{x 4} \vee \neg p_{y 6} & \neg p_{x 5} \vee \neg p_{y 3} & \neg p_{x 5} \vee \neg p_{y 4} & \neg p_{x 5} \vee \neg p_{y 5} & \neg p_{x 5} \vee \neg p_{y 6} \\
\neg p_{x 6} \vee \neg p_{y 2} & \neg p_{x 6} \vee \neg p_{y 3} & \neg p_{x 6} \vee \neg p_{y 4} & \neg p_{x 6} \vee \neg p_{y 5} & \neg p_{x 6} \vee \neg p_{y 6}
\end{array}
$$

Support encoding: The support encoding [8] uses the same Boolean variables and axiom clauses with the direct encoding.

Constraints are encoded into clauses representing supports. When $y=j_{1}, \ldots, y=$ $j_{n}$ is the support of $x=i$, the following clause is added.

$$
\neg p_{x i} \vee p_{y j_{1}} \vee \cdots \vee p_{y j_{n}} .
$$

Therefore, the following 8 clauses are used for $x+y \leq 7$.

$$
\begin{aligned}
& \neg p_{x 2} \vee p_{y 2} \vee p_{y 3} \vee p_{y 4} \vee p_{y 5} \\
& \neg p_{x 3} \vee p_{y 2} \vee p_{y 3} \vee p_{y 4} \\
& \neg p_{x 4} \vee p_{y 2} \vee p_{y 3} \\
& \neg p_{x 5} \vee p_{y 2} \\
& \neg p_{y 2} \vee p_{x 2} \vee p_{x 3} \vee p_{x 4} \vee p_{x 5} \\
& \neg p_{y 3} \vee p_{x 2} \vee p_{x 3} \vee p_{x 4} \\
& \neg p_{y 4} \vee p_{x 2} \vee p_{x 3} \\
& \neg p_{y 5} \vee p_{x 2}
\end{aligned}
$$

Order encoding: The order encoding uses Boolean variables $p_{x i}$ meaning $x \leq i$ for each CSP variable $x$ and each integer constant $i(\ell(x)-1 \leq i \leq u(x))$. Therefore, the following 12 Boolean variables are used to encode the example.

$$
\begin{array}{llllll}
p_{x 1} & p_{x 2} & p_{x 3} & p_{x 4} & p_{x 5} & p_{x 6} \\
p_{y 1} & p_{y 2} & p_{y 3} & p_{y 4} & p_{y 5} & p_{y 6}
\end{array}
$$

The following bound and order clauses are used as axioms for each CSP variable $x$.

$$
\begin{aligned}
& \neg p_{x \ell(x)-1} \\
& \quad p_{x u(x)} \\
& \neg p_{x i-1} \vee p_{x i} \quad(\ell(x) \leq i \leq u(x))
\end{aligned}
$$


Therefore, the following 14 clauses are required.

$$
\begin{gathered}
\neg p_{x 1} \vee p_{x 2} \neg p_{x 2} \vee p_{x 3} \neg p_{x 3} \vee p_{x 4} p_{x 6} \\
\text { (similar clauses for } y)
\end{gathered}
$$

Constraints are encoded into clauses representing conflict regions instead of conflict points. When all points $\left(x_{1}, \ldots, x_{n}\right)$ in the region $i_{1}<x_{1} \leq j_{1}, \ldots, i_{n}<x_{n} \leq$ $j_{n}$ violate the constraint, the following clause is added.

$$
p_{x_{1} i_{1}} \vee \neg p_{x_{1} j_{1}} \vee \cdots \vee p_{x_{n} i_{n}} \vee \neg p_{x_{n} j_{n}}
$$

Therefore, the following 5 clauses are used to encode $x+y \leq 7$.

$$
p_{x 1} \vee p_{y 5} \quad p_{x 2} \vee p_{y 4} \quad p_{x 3} \vee p_{y 3} \quad p_{x 4} \vee p_{y 2} \quad p_{x 5} \vee p_{y 1}
$$

\section{Encoding finite linear COP to SAT}

Definition 4 (Finite linear COP) A (finite linear) COP (Constraint Optimization Problem) is defined as a tuple $(V, \ell, u, B, S, v)$ where

(1) $(V, \ell, u, B, S)$ is a finite linear CSP, and

(2) $v \in V$ is an integer variable representing the objective variable to be minimized (without loss of generality we assume COPs as minimization problems).

The optimal value of COP $(V, \ell, u, B, S, v)$ can be obtained by repeatedly solving CSPs.

$\min \{c \mid \ell(v) \leq c \leq u(v), \operatorname{CSP}(V, \ell, u, B, S \cup\{\{v \leq c\}\})$ is satisfiable $\}$

Of course, instead of linear search, binary search method is useful to find the optimal value efficiently as used in previous works [11, 19, 21].

It is also possible to encode COP to SAT once at first, and repeatedly modify only the clause $\{v \leq c\}$ for a given $c$. This procedure substantially reduces the time spent for encoding.

\section{Experimental results}

In order to show the applicability of our method, we applied it to the Graph Coloring Problems and the Open-Shop Scheduling (OSS) Problems.

\subsection{Graph coloring problems}

Graph Coloring Problem (GCP) is a problem to find the minimum number of colors (the chromatic number) for a given undirected graph such that no two adjacent vertices have the same color. 


\begin{tabular}{|c|c|c|c|c|c|c|}
\hline \multirow{2}{*}{$\begin{array}{l}\text { Fig. } 3 \text { Comparison of MiniSat } \\
\text { (CPU seconds) for } 44 \mathrm{GCP} \\
\text { instances (a) }\end{array}$} & \multirow{3}{*}{$\begin{array}{l}\text { Instance } \\
\text { DSJC125.1 }\end{array}$} & \multicolumn{2}{|c|}{ Colors } & \multirow{2}{*}{$\begin{array}{r}\begin{array}{r}\text { Order } \\
\text { Encoding }\end{array} \\
0.02\end{array}$} & \multirow{2}{*}{$\begin{array}{r}\begin{array}{r}\text { Direct } \\
\text { Encoding }\end{array} \\
0.01\end{array}$} & \multirow{3}{*}{$\begin{array}{r}\begin{array}{r}\text { Support } \\
\text { Encoding }\end{array} \\
0.01\end{array}$} \\
\hline & & 4 & $\mathrm{U}$ & & & \\
\hline & & 5 & $\mathrm{~S}$ & 0.28 & 0.01 & \\
\hline & DSJR500.1 & 11 & $\mathrm{U}$ & 223.86 & & \\
\hline & & 12 & $\mathrm{~S}$ & 0.24 & 0.13 & 0.95 \\
\hline & le450_5a & 4 & $\mathrm{U}$ & 0.16 & 0.04 & 0.08 \\
\hline & & 5 & $\mathrm{~S}$ & 1.63 & 0.20 & 1.05 \\
\hline & le450_5c & 4 & $\mathrm{U}$ & 0.24 & 0.02 & 0.26 \\
\hline & & 5 & $\mathrm{~S}$ & 0.47 & 0.09 & 0.21 \\
\hline & le450_5d & 4 & $\mathrm{U}$ & 0.09 & 0.06 & 0.26 \\
\hline & & 5 & $\mathrm{~S}$ & 0.32 & 0.08 & 0.47 \\
\hline & anna & 10 & $\mathrm{U}$ & 2.16 & 301.25 & 1238.57 \\
\hline & & 11 & $\mathrm{~S}$ & 0.02 & 0.00 & 0.04 \\
\hline & david & 10 & $\mathrm{U}$ & 2.11 & 174.40 & 1359.68 \\
\hline & & 11 & S & 0.02 & 0.01 & 0.04 \\
\hline & homer & 12 & $\mathrm{U}$ & 106.73 & - & - \\
\hline & & 13 & $\mathrm{~S}$ & 0.08 & - & - \\
\hline & huck & 10 & $\mathrm{U}$ & 1.18 & 139.06 & 1189.80 \\
\hline & & 11 & $\mathrm{~S}$ & 0.02 & 0.00 & 0.01 \\
\hline & jean & 9 & U & 0.22 & 15.68 & 36.83 \\
\hline & & 10 & S & 0.00 & 0.01 & 0.01 \\
\hline & games120 & 8 & $\mathrm{U}$ & 0.10 & 3.31 & 7.75 \\
\hline & & 9 & $\mathrm{~S}$ & 0.04 & 0.01 & 0.03 \\
\hline & miles 250 & 7 & $\mathrm{U}$ & 0.04 & 0.11 & 0.53 \\
\hline & & 8 & $\mathrm{~S}$ & 0.01 & 0.01 & 0.04 \\
\hline & queen5_5 & 4 & $\mathrm{U}$ & 0.00 & 0.00 & 0.00 \\
\hline & & 5 & $\mathrm{~S}$ & 0.00 & 0.00 & 0.00 \\
\hline & queen6_6 & 6 & $\mathrm{U}$ & 4.86 & 1.37 & 6.42 \\
\hline & & 7 & $\mathrm{~S}$ & 0.01 & 0.01 & 0.10 \\
\hline & queen7_7 & 6 & $\mathrm{U}$ & 0.02 & 0.01 & 0.04 \\
\hline & & 7 & $\mathrm{~S}$ & 0.01 & 0.00 & 0.01 \\
\hline & queen8_12 & 11 & $\mathrm{U}$ & 26.27 & - & - \\
\hline & & 12 & $\mathrm{~S}$ & 0.03 & 0.03 & 0.28 \\
\hline & myciel3 & 3 & $\mathrm{U}$ & 0.00 & 0.00 & 0.00 \\
\hline & & 4 & $\mathrm{~S}$ & 0.00 & 0.00 & 0.00 \\
\hline & myciel4 & 4 & $\mathrm{U}$ & 0.04 & 0.04 & 0.05 \\
\hline & & 5 & $\mathrm{~S}$ & 0.00 & 0.00 & 0.00 \\
\hline & myciel5 & 5 & $\mathrm{U}$ & 57.30 & 273.22 & 570.50 \\
\hline & & 6 & $\mathrm{~S}$ & 0.00 & 0.00 & 0.00 \\
\hline & mug88_1 & 3 & $\mathrm{U}$ & 0.01 & 0.00 & 0.01 \\
\hline & & 4 & $\mathrm{~S}$ & 0.00 & 0.00 & 0.00 \\
\hline & mug88_25 & 3 & $\mathrm{U}$ & 0.01 & 0.01 & 0.01 \\
\hline & & 4 & S & 0.00 & 0.00 & 0.00 \\
\hline & mug100_1 & 3 & $\mathrm{U}$ & 0.01 & 0.00 & 0.01 \\
\hline & & 4 & $\mathrm{~S}$ & 0.00 & 0.00 & 0.00 \\
\hline
\end{tabular}

All of 119 benchmark instances listed in the web site of the Computational Symposium on Graph Coloring ${ }^{1}$ are used to compare the order encoding with the direct encoding [24] and the support encoding [8].

The encoding programs are written in Perl and MiniSat [6] is used as the SAT solver. These programs find the minimum chromatic number by changing the number of colors with binary search method.

The order encoding program decided the minimum chromatic number of 44 instances within the time limit of $1800 \mathrm{~s}$ executed on Intel Xeon $2.8 \mathrm{GHz} 4 \mathrm{~GB}$ memory machine, while the direct and support encoding programs solved 39 and 38 instances respectively which are included in the 44 instances.

\footnotetext{
${ }^{1}$ http://mat.gsia.cmu.edu/COLOR04/

空 Springer
} 
Fig. 4 Comparison of MiniSat (CPU seconds) for $44 \mathrm{GCP}$ instances (b)

\begin{tabular}{|c|c|c|c|c|c|}
\hline \multirow{2}{*}{$\begin{array}{l}\text { Instance } \\
\text { mug100_25 }\end{array}$} & \multicolumn{2}{|c|}{ Colors } & \multirow{2}{*}{$\begin{array}{r}\begin{array}{r}\text { Order } \\
\text { Encoding }\end{array} \\
0.01\end{array}$} & \multirow{2}{*}{$\begin{array}{r}\begin{array}{r}\text { Direct } \\
\text { Encoding }\end{array} \\
0.01\end{array}$} & \multirow{2}{*}{$\begin{array}{r}\begin{array}{r}\text { Support } \\
\text { Encoding }\end{array} \\
0.01\end{array}$} \\
\hline & 3 & $\mathrm{U}$ & & & \\
\hline & 4 & $S$ & 0.00 & 0.00 & 0.00 \\
\hline \multirow[t]{2}{*}{ abb313GPIA } & 8 & $\mathrm{U}$ & 649.23 & - & - \\
\hline & 9 & $\mathrm{~S}$ & 19.06 & - & \\
\hline \multirow[t]{2}{*}{ ash331GPIA } & 3 & $\mathrm{U}$ & 0.08 & 0.01 & 0.02 \\
\hline & 4 & $\mathrm{~S}$ & 0.03 & 0.01 & 0.07 \\
\hline \multirow[t]{2}{*}{ ash608GPIA } & 3 & $\mathrm{U}$ & 0.20 & 0.01 & 0.11 \\
\hline & 4 & S & 0.09 & 0.06 & 0.16 \\
\hline \multirow[t]{2}{*}{ ash958GPIA } & 3 & $\mathrm{U}$ & 0.16 & 0.03 & 0.17 \\
\hline & 4 & $\mathrm{~S}$ & 0.44 & 0.08 & 0.24 \\
\hline \multirow[t]{2}{*}{ will199GPIA } & 6 & $\mathrm{U}$ & 0.18 & 0.16 & 0.69 \\
\hline & 7 & $\mathrm{~S}$ & 0.18 & 0.10 & 0.27 \\
\hline \multirow[t]{2}{*}{ 1-Insertions_4 } & 4 & $\mathrm{U}$ & 131.34 & 403.12 & 881.53 \\
\hline & 5 & $\mathrm{~S}$ & 0.00 & 0.00 & 0.00 \\
\hline \multirow[t]{2}{*}{ 2-Insertions_3 } & 3 & $\mathrm{U}$ & 0.04 & 0.02 & 0.03 \\
\hline & 4 & S & 0.00 & 0.00 & 0.00 \\
\hline \multirow[t]{2}{*}{ 3-Insertions_3 } & 3 & $\mathrm{U}$ & 0.24 & 0.11 & 0.22 \\
\hline & 4 & $\mathrm{~S}$ & 0.00 & 0.00 & 0.01 \\
\hline \multirow[t]{2}{*}{ 4-Insertions_3 } & 3 & $\mathrm{U}$ & 1.80 & 1.62 & 2.42 \\
\hline & 4 & S & 0.00 & 0.00 & 0.00 \\
\hline \multirow[t]{2}{*}{ 1-FullIns_3 } & 3 & $\mathrm{U}$ & 0.00 & 0.00 & 0.00 \\
\hline & 4 & $\mathrm{~S}$ & 0.00 & 0.00 & 0.00 \\
\hline \multirow[t]{2}{*}{ 1-FullIns_4 } & 4 & $\mathrm{U}$ & 0.03 & 0.01 & 0.03 \\
\hline & 5 & $\mathrm{~S}$ & 0.02 & 0.00 & 0.02 \\
\hline \multirow[t]{2}{*}{ 1-FullIns_5 } & 5 & $\mathrm{U}$ & 10.46 & 15.34 & 60.34 \\
\hline & 6 & S & 0.04 & 0.01 & 0.12 \\
\hline \multirow[t]{2}{*}{ 2-FullIns_3 } & 4 & $\mathrm{U}$ & 0.00 & 0.00 & 0.00 \\
\hline & 5 & S & 0.00 & 0.00 & 0.01 \\
\hline \multirow[t]{2}{*}{ 2-FullIns_4 } & 5 & $\mathrm{U}$ & 0.14 & 0.10 & 0.39 \\
\hline & 6 & $\mathrm{~S}$ & 0.03 & 0.01 & 0.03 \\
\hline \multirow[t]{2}{*}{ 2-FullIns_5 } & 6 & $\mathrm{U}$ & 217.55 & 999.09 & - \\
\hline & 7 & S & 0.62 & 0.04 & - \\
\hline \multirow[t]{2}{*}{ 3-FullIns_3 } & 5 & $\mathrm{U}$ & 0.02 & 0.01 & 0.02 \\
\hline & 6 & $\mathrm{~S}$ & 0.02 & 0.00 & 0.02 \\
\hline \multirow[t]{2}{*}{ 3-FullIns_4 } & 6 & $\mathrm{U}$ & 1.48 & 3.87 & 14.96 \\
\hline & 7 & S & 0.16 & 0.05 & 0.13 \\
\hline \multirow[t]{2}{*}{ 4-FullIns_3 } & 6 & $\mathrm{U}$ & 0.06 & 0.05 & 0.12 \\
\hline & 7 & $\mathrm{~S}$ & 0.00 & 0.00 & 0.02 \\
\hline \multirow[t]{2}{*}{ 4-FullIns_4 } & 7 & $\mathrm{U}$ & 17.47 & 192.58 & 314.54 \\
\hline & 8 & $\mathrm{~S}$ & 0.19 & 0.04 & 0.31 \\
\hline 5-FullIns_3 & 7 & $\mathrm{U}$ & 0.23 & 0.68 & 4.46 \\
\hline & 8 & $\mathrm{~S}$ & 0.01 & 0.01 & 0.02 \\
\hline 5-FullIns_4 & 8 & $\mathrm{U}$ & 277.85 & - & \\
\hline & 9 & $\mathrm{~S}$ & 0.69 & 0.22 & 0.33 \\
\hline
\end{tabular}

Figures 3 and 4 list the solved instances for each encoding method along with the CPU seconds of MiniSat solver for the SAT problem with the optimum chromatic number $c$ (followed by a mark of "S") and the SAT problem with $c-1$ colors (followed by a mark of "U”). For example, the instance DSJC125.1 can be colored with five colors but not with four colors.

The result shows the order encoding is also effective for CSPs with not-equals constraints.

Fig. 5 OSS benchmark instance gp03-01

$$
\left(p_{i j}\right)=\left(\begin{array}{rrr}
661 & 6 & 333 \\
168 & 489 & 343 \\
171 & 505 & 324
\end{array}\right)
$$




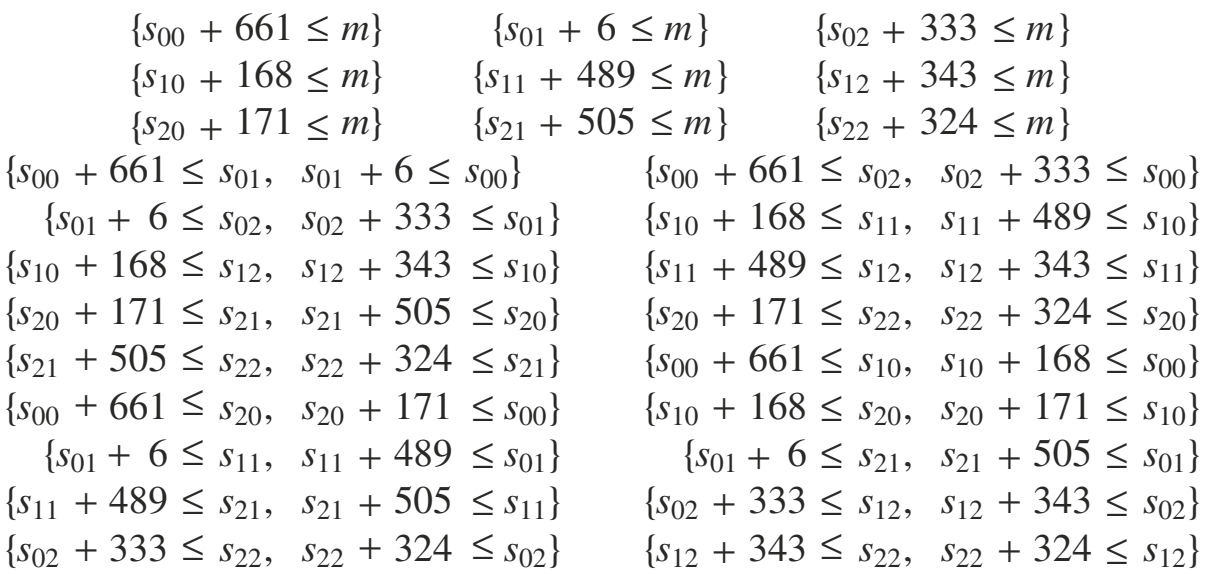

Fig. 6 CSP representation of gp03-01

\subsection{Open-shop scheduling problems}

There are three well-known sets of OSS (Open-Shop Scheduling) benchmark problems by Guéret and Prins [9] (80 instances denoted by gp*), Taillard [22] (60 instances denoted by tai_*), and Brucker et al. [3] (52 instances denoted by $j *)$, which are also used in $[2,13,15]$.

Some problems in these benchmark sets are very hard to solve. Actually, three instances (j7-per0-0, j8-per0-1, and j8-per10-2) are still open, and 37 instances are closed recently in 2005 by complete MCS-based search solver of ILOG [15].

Representing OSS problem as CSP is straightforward. Figure 5 defines a benchmark instance gp03-01 of 3 jobs and 3 machines. Each element $p_{i j}$ represents the process time of the operation $O_{i j}(0 \leq i, j \leq 2)$. The instance gp03-01 can be represented as a CSP of 27 clauses as shown in Fig. 6.

In the figure, integer variables $m$ represents the makespan and each $s_{i j}$ represents the start time of each operation $O_{i j}$. Clauses $\left\{s_{i j}+p_{i j} \leq m\right\}$ represent deadline constraint such that operations should be completed before $m$. Clauses $\left\{s_{i j}+p_{i j} \leq s_{k l}\right.$, $\left.s_{k l}+p_{k l} \leq s_{i j}\right\}$ represent resource capacity constraint such that the operation $O_{i j}$ and $O_{k l}$ should not be overlapped each other.

Fig. 7 Number of solved instances within a specified CPU time

\begin{tabular}{rrr}
\hline \multicolumn{2}{c}{ CPU time } & Number of solved instances \\
\hline 0 min. .. & 1 min. & 96 \\
1 min. .. & 10 min. & 77 \\
10 min. .. & 1 hour & 14 \\
1 hour .. & 3 hours & 3 \\
\multicolumn{2}{c}{ Unsolved } & 2 \\
\hline \multicolumn{2}{c}{ Total } & 192 \\
\hline
\end{tabular}


Fig. 8 Optimal scheduling of j8-per10-2 found by CSP2SAT

$$
\left(s_{i j}\right)=\left(\begin{array}{rrrrrrrr}
247 & 296 & 110 & 618 & 537 & 31 & 500 & 127 \\
815 & 50 & 328 & 274 & 311 & 672 & 550 & 6 \\
1 & 583 & 120 & 339 & 876 & 842 & 675 & 58 \\
293 & 669 & 5 & 72 & 250 & 502 & 403 & 994 \\
286 & 517 & 870 & 594 & 612 & 347 & 0 & 297 \\
404 & 252 & 73 & 28 & 83 & 25 & 300 & 734 \\
707 & 997 & 560 & 12 & 48 & 87 & 842 & 340 \\
53 & 6 & 703 & 285 & 342 & 872 & 526 & 547
\end{array}\right)
$$

Before encoding the CSP to SAT, we also need to determine the lower and upper bound of integer variables. We used the following values $\ell$ and $u$ (where $n$ is the number of jobs and machines).

$$
\begin{aligned}
& \ell=\max \left(\max _{0 \leq i<n} \sum_{0 \leq j<n} p_{i j}, \max _{0 \leq j<n} \sum_{0 \leq i<n} p_{i j}\right) \\
& u=\sum_{0 \leq k<n} \max _{(i-j) \bmod n=k} p_{i j}
\end{aligned}
$$

The value $u$ is used for the upper bound of $s_{i j}$ 's and $m$, and the value $\ell$ is used for the lower bound of $m$ (the lower bound 0 is used for $s_{i j}$ 's). For example, $\ell=1000$ and $u=1509$ for the instance gp03-01.

We developed a program called CSP2SAT which encodes a CSP representation (of a given OSS problem) into SAT and repeatedly invokes a complete SAT solver to find the optimal solution by binary search. ${ }^{2}$ We used MiniSat [6] as the backend complete SAT solver because it is known to be very efficient.

We run CSP2SAT for all 192 instances of the three benchmark sets on Intel Xeon 2.8 GHz 4 GB memory machine with the time limit of $3 \mathrm{~h}$ (10800 s).

Figures 12, 13, and 14 provides the results for each benchmark instance. The column named "Optim." describes the optimal value found by the program, and "CPU" describes the total CPU time in seconds including encoding process. The column named "SAT" describes the numbers of Boolean variables and clauses in the encoded SAT problem. Although time spent for encoding is not shown separately in the figures, it ranges from $1 \mathrm{~s}$ to $1163 \mathrm{~s}$ and fits linearly with the number of clauses in the encoded SAT program.

CSP2SAT found the optimal solutions for 189 known problems and one unknown problem (j8-per10-2) within $3 \mathrm{~h}$. Figure 7 shows the overall performance. 96 instances $(50 \%)$ are solved within $1 \mathrm{~min}$, and 173 instances $(90 \%)$ are solved within $10 \mathrm{~min}$.

The known upper bound of j 8-per10-2 was 1009. CSP2SAT improved the result to 1002 and proved there are no solutions for 1001. Figure 8 shows the start times $s_{i j}$ of the optimal scheduling found by the program.

Figure 9 provides the log scale plot of the number of clauses in the encoded SAT problem ( $x$-axis) and the total CPU time ( $y$-axis) for 190 problems. The mark + is

${ }^{2}$ The program will be available at http://bach.istc.kobe-u.ac.jp/csp2sat/. 


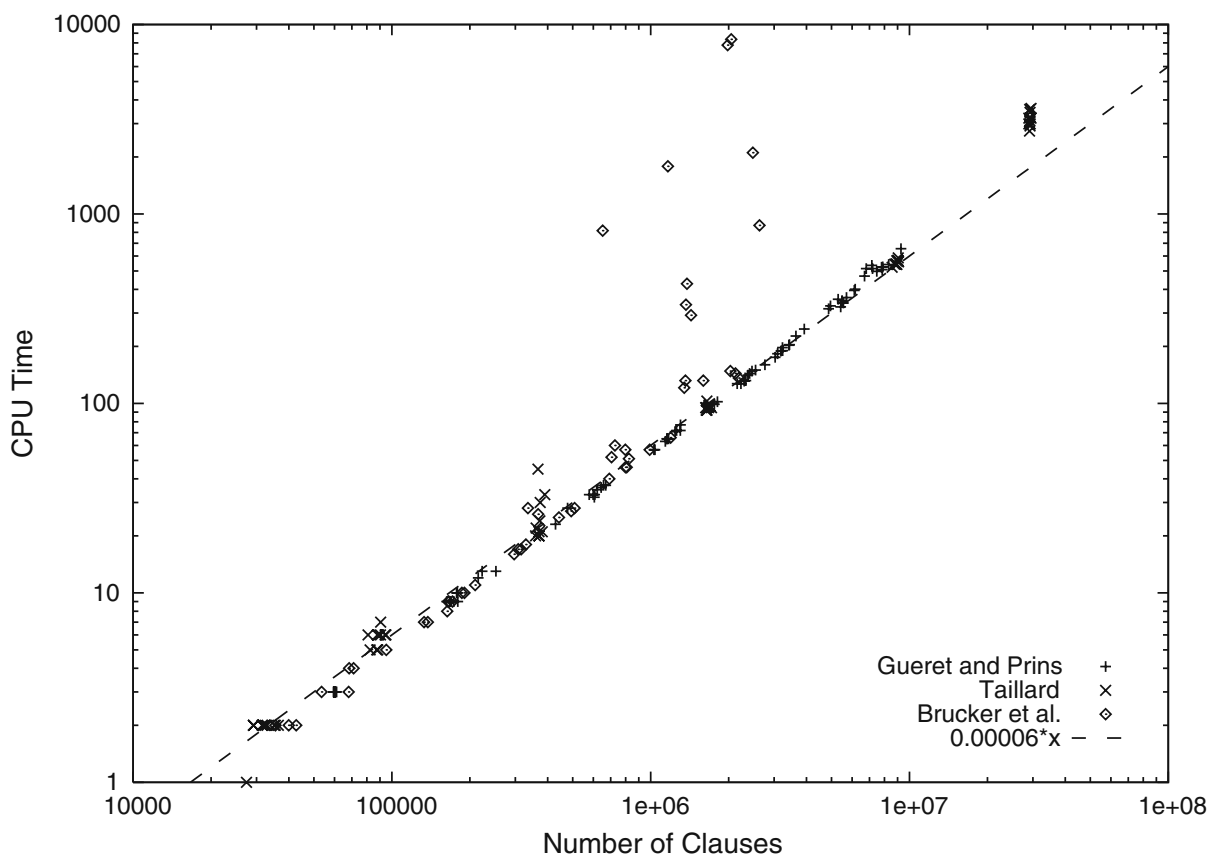

Fig. 9 Log scale plot of the number of clauses and the CPU time (seconds)

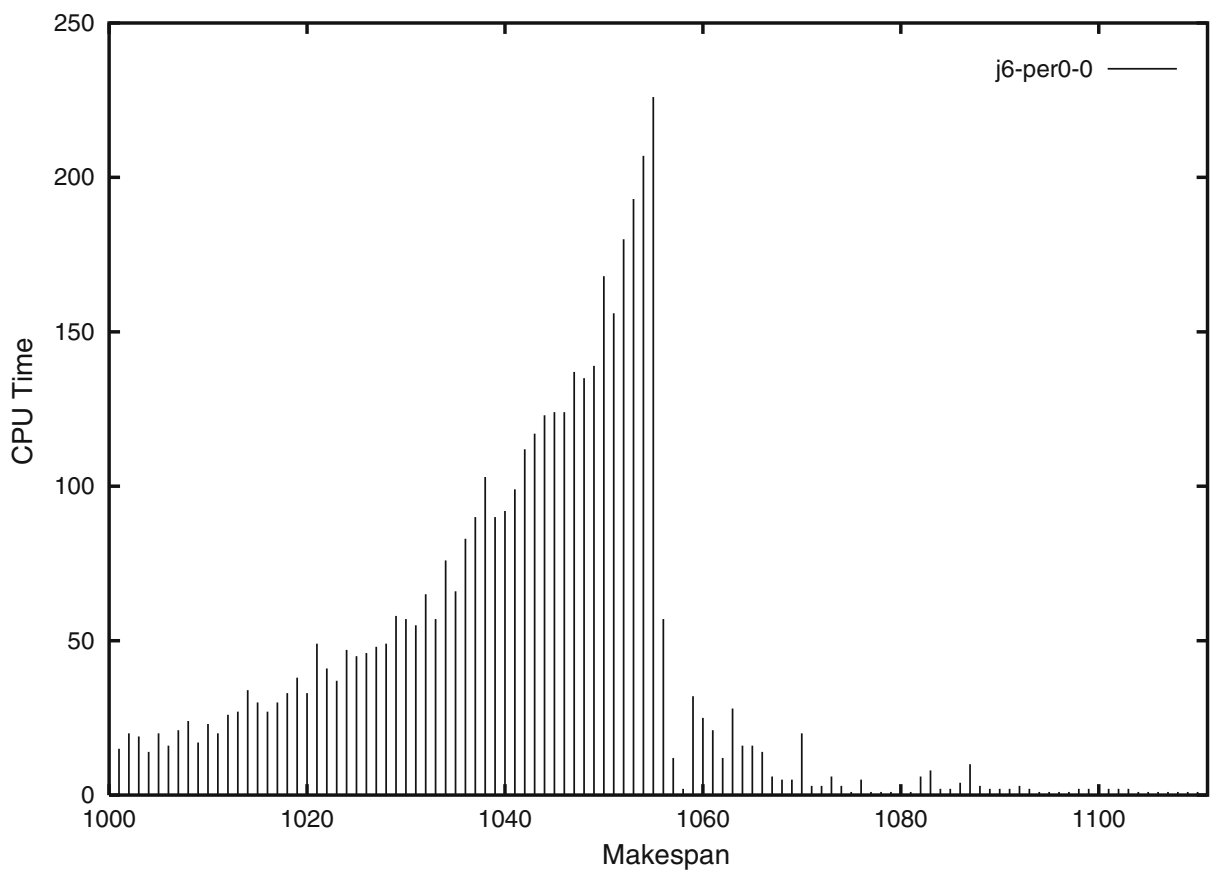

Fig. $10 \mathrm{CPU}$ seconds of checking satisfiability for various makespan values Springer 
Fig. 11 New results found and proved to be optimal

\begin{tabular}{lrrr}
\hline Instance & Makespan & \multicolumn{2}{c}{ Previously known bounds } \\
\cline { 3 - 4 } & & Lower bound & Upper \\
\hline j7-pound \\
j8-per0-0 & 1048 & 1039 & 1048 \\
j8-per10-2 & 1039 & 1018 & 1039 \\
\hline
\end{tabular}

used for gp* benchmarks, $\times$ is used for tai* benchmarks, and $\diamond$ is used for $j$ * benchmarks. Dotted line is a plot of $y=0.00006 x$.

Except some instances of $j *$ benchmarks, it seems the total CPU time linearly fits with the number of clauses. This shows that the encoding used in this paper is natural and does not uselessly increase the complexity for SAT solver.

\begin{tabular}{lrrrr}
\hline Instance Optim. & CPU & \multicolumn{2}{c}{ SAT } \\
\cline { 4 - 5 } & & & Variables & Clauses \\
\hline gp03-01 & 1168 & 3 & 14155 & 61133 \\
gp03-02 & 1170 & 3 & 13945 & 59978 \\
gp03-03 & 1168 & 3 & 13945 & 59978 \\
gp03-04 & 1166 & 3 & 13995 & 60253 \\
gp03-05 & 1170 & 3 & 13855 & 59483 \\
gp03-06 & 1169 & 3 & 13915 & 59813 \\
gp03-07 & 1165 & 3 & 13925 & 59868 \\
gp03-08 & 1167 & 3 & 13955 & 60033 \\
gp03-09 & 1162 & 3 & 14075 & 60693 \\
gp03-10 & 1165 & 3 & 13945 & 59978 \\
gp04-01 & 1281 & 10 & 28097 & 179010 \\
gp04-02 & 1270 & 13 & 33928 & 223257 \\
gp04-03 & 1288 & 9 & 28182 & 179655 \\
gp04-04 & 1261 & 12 & 32925 & 215646 \\
gp04-05 & 1289 & 10 & 27927 & 177720 \\
gp04-06 & 1269 & 9 & 27383 & 173592 \\
gp04-07 & 1267 & 9 & 25955 & 162756 \\
gp04-08 & 1259 & 9 & 26516 & 167013 \\
gp04-09 & 1280 & 9 & 26737 & 168690 \\
gp04-10 & 1263 & 13 & 37736 & 252153 \\
gp05-01 & 1245 & 36 & 72727 & 643703 \\
gp05-02 & 1247 & 33 & 65993 & 578694 \\
gp05-03 & 1265 & 37 & 75457 & 670058 \\
gp05-04 & 1258 & 23 & 50497 & 429098 \\
gp05-05 & 1280 & 33 & 68151 & 599527 \\
gp05-06 & 1269 & 37 & 74131 & 657257 \\
gp05-07 & 1269 & 32 & 68801 & 605802 \\
gp05-08 & 1287 & 28 & 55489 & 477290 \\
gp05-09 & 1262 & 35 & 70387 & 621113 \\
gp05-10 & 1254 & 33 & 69009 & 607810 \\
gp06-01 & 1264 & 57 & 96410 & 1038543 \\
gp06-02 & 1285 & 65 & 106659 & 1158484 \\
gp06-03 & 1255 & 72 & 115317 & 1259806 \\
gp06-04 & 1275 & 63 & 104957 & 1138566 \\
gp06-05 & 1299 & 65 & 107806 & 1171907 \\
gp06-06 & 1284 & 65 & 106400 & 1155453 \\
\hline gp06-09 & 1290 & 77 & 119091 & 1303972 \\
& 1265 & 71 & 113726 & 1241187 \\
& 1254 & 57 & 95559 & 1028584 \\
\hline
\end{tabular}

\begin{tabular}{|c|c|c|c|c|}
\hline \multicolumn{2}{|c|}{ Instance Optim. } & \multirow[t]{2}{*}{ CPU } & \multicolumn{2}{|c|}{ SAT } \\
\hline & & & Variables & Clauses \\
\hline gp07-01 & 1159 & 99 & 137537 & 1761090 \\
\hline gp07-02 & 1185 & 148 & 188537 & 2461830 \\
\hline gp07-03 & 1237 & 132 & 179037 & 2331300 \\
\hline gp07-04 & 1167 & 131 & 176437 & 2295576 \\
\hline gp07-05 & 1157 & 141 & 182137 & 2373894 \\
\hline gp07-06 & 1193 & 127 & 166587 & 2160237 \\
\hline gp07-07 & 1185 & 102 & 141187 & 1811241 \\
\hline gp07-08 & 1180 & 144 & 184787 & 2410305 \\
\hline gp07-09 & 1220 & 150 & 437 & 2542896 \\
\hline gp07-10 & 1270 & 127 & 171837 & 2232372 \\
\hline gp08-01 & 1130 & 160 & 186315 & 2762188 \\
\hline gp08-02 & 1135 & 190 & 215 & 3233688 \\
\hline gp08-03 & 1110 & 197 & 215955 & 3229588 \\
\hline gp08-04 & 1153 & 227 & 242020 & 3640613 \\
\hline gp08-05 & 1218 & 247 & 830 & 3921463 \\
\hline gp08-06 & 1115 & 175 & 203085 & 3026638 \\
\hline gp08-07 & 1126 & 204 & 229215 & 3438688 \\
\hline gp08-08 & 1148 & 183 & 245 & 3092 \\
\hline gp08-09 & 1114 & 189 & 213225 & 3186538 \\
\hline gp08-10 & 1161 & 203 & 227980 & 3419213 \\
\hline gp09-01 & 1129 & 323 & 881 & 5423 \\
\hline gp09-02 & 1110 & 327 & 477 & 4954180 \\
\hline gp09-03 & 1115 & 395 & 357077 & 6121380 \\
\hline gp09-04 & 1130 & 340 & 2063 & 5498387 \\
\hline gp09-05 & 1180 & 362 & 871 & 5708483 \\
\hline gp09-06 & 1093 & 401 & 359455 & 6163691 \\
\hline gp09-07 & 1090 & 339 & 325507 & 5559665 \\
\hline gp09-08 & 1105 & 349 & 321325 & 5485256 \\
\hline gp09-09 & 1123 & 316 & 286803 & 4871017 \\
\hline gp09-10 & 1110 & 355 & 310993 & 5301422 \\
\hline gp 10-01 & & 470 & 353491 & 6705492 \\
\hline gp $10-02$ & 1097 & 526 & 412677 & 7878078 \\
\hline gp $10-03$ & 1081 & 535 & 376317 & 7157718 \\
\hline gp 10-04 & 1077 & 515 & 378438 & 7199739 \\
\hline gp $10-05$ & 1071 & 515 & 358743 & 6809544 \\
\hline gp 10-06 & 1071 & 508 & 410960 & 7844061 \\
\hline gp 10-07 & 1079 & 523 & 408839 & 7802040 \\
\hline gp $10-08$ & 1093 & 498 & 392578 & 7479879 \\
\hline gp 10-09 & 1112 & 541 & 434897 & 8318298 \\
\hline gp 10-10 & 1092 & 656 & 483276 & 9276777 \\
\hline
\end{tabular}

Fig. 12 Results for benchmark instances provided by Guéret and Prins 


\begin{tabular}{|c|c|c|c|c|}
\hline \multirow[t]{2}{*}{ Instance } & \multirow[t]{2}{*}{ Optim. } & \multirow[t]{2}{*}{$\mathrm{CPU}$} & \multicolumn{2}{|c|}{ SAT } \\
\hline & & & Variables & Clauses \\
\hline tai_4x4_1 & 193 & 2 & 5043 & 31706 \\
\hline tai_4x4_2 & 236 & 1 & 4643 & 27426 \\
\hline tai_4x4_3 & 271 & 2 & 5460 & 32925 \\
\hline tai_4x4_4 & 250 & 2 & 5358 & 32341 \\
\hline tai_4x4_5 & 295 & 2 & 6081 & 36418 \\
\hline tai_4x4_6 & 189 & 2 & 4721 & 29194 \\
\hline tai_4x4_7 & 201 & 2 & 4743 & 29188 \\
\hline tai_4x4_8 & 217 & 2 & 5629 & 35110 \\
\hline tai_4x4_9 & 261 & & 5328 & 31517 \\
\hline x4_10 & 217 & & 5611 & 35444 \\
\hline tai_5x5_1 & 300 & 6 & 11526 & 94098 \\
\hline tai_5x5_2 & 262 & 5 & 0110 & 82314 \\
\hline tai_5x5_3 & 323 & 6 & 11318 & 90297 \\
\hline tai_5x5_4 & 310 & 5 & 11047 & 88190 \\
\hline tai_5x5_5 & 326 & 6 & 0356 & 80906 \\
\hline tai_5x5_6 & 312 & 5 & 10942 & 87344 \\
\hline tai_-5x5_7 & 303 & 6 & 10951 & 87906 \\
\hline tai_ $5 \times 5$ × 8 & 300 & 6 & 11009 & 88852 \\
\hline tai_5x5_9 & 353 & 6 & 11940 & 94884 \\
\hline$-5 \times 5 \_10$ & 326 & 7 & 11344 & 90508 \\
\hline tai_7x7_1 & 435 & 21 & 30952 & 370295 \\
\hline tai__7x7_2 & 443 & 24 & 31244 & 372853 \\
\hline tai_7x7_3 & 468 & 30 & 31669 & 374258 \\
\hline tai_7x7_4 & 463 & 20 & 31224 & 370305 \\
\hline tai_7x7_5 & 416 & 22 & 30171 & 360661 \\
\hline$x 7 \_6$ & 451 & 45 & 30986 & 367026 \\
\hline x7_7 & 422 & 33 & 32415 & 389596 \\
\hline x7_8 & 424 & 20 & 30863 & 370287 \\
\hline i_7x7_9 & 458 & 21 & 31929 & 380761 \\
\hline tai_ $7 \times 7-10$ & 398 & 20 & 29939 & 359194 \\
\hline
\end{tabular}

\begin{tabular}{|c|c|c|c|c|}
\hline \multirow[t]{2}{*}{ Instance } & \multirow[t]{2}{*}{ Optim. } & \multirow[t]{2}{*}{$\mathrm{CPU}$} & \multicolumn{2}{|c|}{ SAT } \\
\hline & & & Variables & Clauses \\
\hline tai_10x10_1 & 637 & 98 & 94183 & 1678890 \\
\hline tai_10x10_2 & 588 & 95 & 95343 & 1716326 \\
\hline tai_10 $10 \times 10 \_3$ & 598 & 92 & 92303 & 1651992 \\
\hline tai_10x10_4 & 577 & 92 & 91314 & 1639647 \\
\hline tai_10x10_5 & 640 & 96 & 93978 & 1677177 \\
\hline tai_10x10_6 & 538 & 95 & 91151 & 1642608 \\
\hline tai_10x10_7 & 616 & 103 & 92285 & 1648788 \\
\hline tai_10x10_8 & 595 & 95 & 91094 & 1631685 \\
\hline tai_10x10_9 & 595 & 97 & 94528 & 1697235 \\
\hline tai_10x10_10 & 596 & 95 & 93315 & 1674220 \\
\hline tai_15x15_1 & 937 & 523 & 309784 & 8563684 \\
\hline tai_15x15_2 & 918 & 567 & 325397 & 9026993 \\
\hline tai_15x15_3 & 871 & 543 & 315726 & 8767426 \\
\hline tai_15x15_4 & 934 & 560 & 326511 & 9067128 \\
\hline tai_15x15_5 & 946 & 541 & 323109 & 8940331 \\
\hline tai_15x15_6 & 933 & 560 & 326512 & 9067214 \\
\hline t ai_15x15_7 & 891 & 566 & 322034 & 8943618 \\
\hline tai_15x15_8 & 893 & 546 & 319320 & 8866998 \\
\hline t ai_15x15_9 & 899 & 568 & 324060 & 8998985 \\
\hline t ai_15x15_10 & 902 & 586 & 325865 & 9053491 \\
\hline t ai_20x20_1 & 1155 & 3105 & 775142 & 29178719 \\
\hline tai_20x20_2 & 1241 & 3559 & 777061 & 29153596 \\
\hline tai_20 20 20_3 & 1257 & 2990 & 770228 & 28898989 \\
\hline tai_20x20_4 & 1248 & 3442 & 779059 & 29238508 \\
\hline tai_20 20 20_5 & 1256 & 3603 & 785066 & 29485803 \\
\hline tai_20x20_6 & 1204 & 2741 & 773489 & 29073596 \\
\hline tai_20x20_7 & 1294 & 2912 & 779414 & 29225385 \\
\hline tai_20x20_8 & 1169 & 2990 & 778336 & 29262619 \\
\hline tai_20x20_9 & 1289 & 3204 & 785835 & 29493666 \\
\hline tai_20x20_10 & 1241 & 3208 & 770645 & 28917758 \\
\hline
\end{tabular}

Fig. 13 Results for benchmark instances provided by Taillard

\begin{tabular}{lrrrr}
\hline Instance & Optim. & CPU & \multicolumn{2}{c}{ SAT } \\
\cline { 3 - 5 } & & & Variables & Clauses \\
\hline j3-per0-1 & 1127 & 2 & 10805 & 42708 \\
j3-per0-2 & 1084 & 5 & 20335 & 95123 \\
j3-per10-0 & 1131 & 3 & 12675 & 53453 \\
j3-per10-1 & 1069 & 3 & 15335 & 68062 \\
j3-per10-2 & 1053 & 4 & 15355 & 68341 \\
j3-per20-0 & 1026 & 2 & 10015 & 39923 \\
j3-per20-1 & 1000 & 2 & 9245 & 35496 \\
j3-per20-2 & 1000 & 4 & 15755 & 71137 \\
j4-per0-0 & 1055 & 7 & 22062 & 133215 \\
j4-per0-1 & 1180 & 11 & 32160 & 209841 \\
j4-per0-2 & 1071 & 8 & 26057 & 163530 \\
j4-per10-0 & 1041 & 10 & 29457 & 190740 \\
j4-per10-1 & 1019 & 7 & 22538 & 137589 \\
j4-per10-2 & 1000 & 9 & 26057 & 164892 \\
j4-per20-0 & 1000 & 10 & 28726 & 186429 \\
j4-per20-1 & 1004 & 9 & 26074 & 165849 \\
j4-per20-2 & 1009 & 9 & 26822 & 171525 \\
j5-per0-0 & 1042 & 28 & 40825 & 335726 \\
j5-per0-1 & 1054 & 28 & 58687 & 508163 \\
j5-per0-2 & 1063 & 26 & 44127 & 367603 \\
j5-per10-0 & 1004 & 18 & 39967 & 329523 \\
j5-per10-1 & 1002 & 17 & 37653 & 307928 \\
j5-per10-2 & 1006 & 16 & 36509 & 296700 \\
j5-per20-0 & 1000 & 17 & 38329 & 315830 \\
j5-per20-1 & 1000 & 27 & 56607 & 492707 \\
j5-per20-2 & 1012 & 25 & 51485 & 442196 \\
\hline
\end{tabular}

\begin{tabular}{lrrrr}
\hline Instance & \multirow{2}{*}{ Optim. } & CPU & \multicolumn{2}{c}{ SAT } \\
\cline { 3 - 5 } & & & Variables & Clauses \\
\hline j6-per0-0 & 1056 & 817 & 63443 & 652740 \\
j6-per0-1 & 1045 & 57 & 92340 & 990913 \\
j6-per0-2 & 1063 & 57 & 75801 & 797362 \\
j6-per10-0 & 1005 & 52 & 67661 & 705462 \\
j6-per10-1 & 1021 & 46 & 76467 & 808206 \\
j6-per10-2 & 1012 & 51 & 77799 & 823964 \\
j6-per20-0 & 1000 & 60 & 69400 & 727773 \\
j6-per20-1 & 1000 & 46 & 75431 & 798740 \\
j6-per20-2 & 1000 & 40 & 66181 & 692002 \\
j7-per0-0 & - & - & 85887 & 1051419 \\
j7-per0-1 & 1055 & 428 & 109837 & 1380492 \\
j7-per0-2 & 1056 & 292 & 113537 & 1431330 \\
j7-per10-0 & 1013 & 332 & 108687 & 1368170 \\
j7-per10-1 & 1000 & 121 & 107087 & 1347411 \\
j7-per10-2 & 1011 & 1786 & 93887 & 1165467 \\
j7-per20-0 & 1000 & 66 & 95487 & 1193523 \\
j7-per20-1 & 1005 & 132 & 125087 & 1595847 \\
j7-per20-2 & 1003 & 132 & 107987 & 1361349 \\
j8-per0-1 & - & - & 145495 & 2118473 \\
j8-per0-2 & 1052 & 870 & 177995 & 2630988 \\
j8-per10-0 & 1017 & 2107 & 168310 & 2481679 \\
j8-per10-1 & 1000 & 8346 & 140620 & 2047787 \\
j8-per10-2 & 1002 & 7789 & 136655 & 1984646 \\
j8-per20-0 & 1000 & 148 & 139255 & 2030756 \\
j8-per20-1 & 1000 & 136 & 149265 & 2191364 \\
j8-per20-2 & 1000 & 144 & 145300 & 2125157 \\
\hline & & & &
\end{tabular}

Fig. 14 Results for benchmark instances provided by Brucker et al. 
We found the most of the CPU time was spent for showing satisfiability and unsatisfiability of the boundary makespan values. Figure 10 plots the CPU seconds spent for checking satisfiability by changing makespan values of the j6-per0-0 instance. The time of $226 \mathrm{~s}$ is required to prove the makespan value 1055 is unsatisfiable, and the time of $57 \mathrm{~s}$ is required to prove 1056 is satisfiable for $j 6$-per $0-0$ which spent $817 \mathrm{~s}$ in total.

For the remaining two open problems $j 7$-per $0-0$ and $j 8$-per $0-1$, we solved and proved their optimal values by using 10 Mac mini machines (PowerPC G4 $1.42 \mathrm{GHz} 1 \mathrm{~GB}$ memory) running in parallel on Xgrid system [1] and by dividing the problem into 120 subproblems where each subproblem is obtained by specifying the order of six operations.

Optimal solutions were found and proved for both of the two remaining instances. The makespan value 1048 is proved to be optimal for the instance j7-per0-0 within $6 \mathrm{~h}$, and the makespan value 1039 is proved to be optimal for the instance j8-per0-1 within $13 \mathrm{~h}$.

Figure 11 summarizes the newly obtained results. All three remaining open problems in $[2,13,15]$ are now closed (Figs. 12, 13, and 14).

\section{Conclusion}

In this paper, we proposed a method to encode Constraint Satisfaction Problems (CSP) and Constraint Optimization Problems (COP) with integer linear constraints into Boolean Satisfiability Testing Problems (SAT).

To evaluate the effectiveness of this approach, we applied the method to the Graph Coloring Problems and the Open-Shop Scheduling Problems (OSS). The proposed method gives the better performance compared with the direct encoding and the support encoding for the Graph Coloring Problems. As for OSS problems, all 192 instances in three OSS benchmark sets are examined, and our program found and proved the optimal results for all instances including three previously undecided problems.

Acknowledgements We would like to give thanks to Katsumi Inoue, Hidetomo Nabeshima, Takehide Soh, and Shuji Ohnishi for their helpful suggestions.

Open Access This article is distributed under the terms of the Creative Commons Attribution Noncommercial License which permits any noncommercial use, distribution, and reproduction in any medium, provided the original author(s) and source are credited.

\section{References}

1. Apple Computer Inc. (2004). Xgrid guide. Cupertino: Apple Computer Inc.

2. Blum, C. (2005). Beam-ACO-hybridizing ant colony optimization with beam search: An application to open shop scheduling. Computers \& OR, 32, 1565-1591.

3. Brucker, P., Hurink, J., Jurisch, B., \& Wöstmann, B. (1997). A branch \& bound algorithm for the open-shop problem. Discrete Applied Mathematics, 76, 43-59.

4. Crawford, J. M., \& Baker, A. B. (1994). Experimental results on the application of satisfiability algorithms to scheduling problems. In Proceedings of the 12th national conference on artificial intelligence (AAAI-94) (pp. 1092-1097). 
5. de Kleer, J. (1989). A comparison of ATMS and CSP techniques. In Proceedings of the 11th international joint conference on artificial intelligence (IJCAI 89) (pp. 290-296).

6. Eén, N., \& Sörensson, N. (2003). An extensible SAT-solver. In Proceedings of the 6th international conference on theory and applications of satisfiability testing (SAT 2003) (pp. 502-518).

7. Ernst, M. D., Millstein, T. D., \& Weld, D. S. (1997). Automatic SAT-compilation of planning problems. In Proceedings of the 15th international joint conference on artificial intelligence (IJCAI 97) (pp. 1169-1177).

8. Gent, I. P. (2002). Arc consistency in SAT. In Proceedings of the 15th european conference on artificial intelligence (ECAI 2002) (pp. 121-125).

9. Guéret, C., \& Prins, C. (1999). A new lower bound for the open-shop problem. Annals of Operations Research, 92, 165-183.

10. Hoos, H. H. (1999). SAT-encodings, search space structure, and local search performance. In Proceedings of the 16th international joint conference on artificial intelligence (IJCAI 99) (pp. 296-303).

11. Inoue, K., Soh, T., Ueda, S., Sasaura, Y., Banbara, M., \& Tamura, N. (2006). A competitive and cooperative approach to propositional satisfiability. Discrete Applied Mathematics, 154, 2291-2306.

12. Iwama, K., \& Miyazaki, S. (1994). SAT-variable complexity of hard combinatorial problems. In Proceedings of the IFIP 13th world computer congress (pp. 253-258).

13. Jussien, N., \& Lhomme, O. (2002). Local search with constraint propagation and conflict-based heuristics. Artificial Intelligence, 139, 21-45.

14. Kautz, H. A., McAllester, D. A., \& Selman, B. (1996). Encoding plans in propositional logic. In Proceedings of the 5th international conference on principles of knowledge representation and reasoning (KR'96) (pp. 374-384).

15. Laborie, P. (2005). Complete MCS-based search: Application to resource constrained project scheduling. In Proceedings of the nineteenth international joint conference on artificial intelligence (IJCAI-05) (pp. 181-186).

16. Li, C. M., \& Anbulagan (1997). Heuristics based on unit propagation for satisfiability problems. In Proceedings of the fifteenth international joint conference on artificial intelligence (IJCAI 97) (pp. 366-371).

17. Marques-Silva, J. P., \& Sakallah, K. A. (1999). GRAPS: A search algorithm for propositional satisfiability. IEEE Transactions on Computers, 48, 506-521.

18. Moskewicz, M. W., Madigan, C. F., Zhao, Y., Zhang, L., \& Malik, S. (2001). Chaff: Engineering an efficient SAT solver. In Proceedings of the 38th design automation conference (DAC 2001) (pp. 530-535).

19. Nabeshima, H., Soh, T., Inoue, K., \& Iwanuma, K. (2006). Lemma reusing for SAT based planning and scheduling. In Proceedings of the international conference on automated planning and scheduling 2006 (ICAPS'06) (pp. 103-112).

20. Selman, B., Kautz, H. A., \& Cohen, B. (1996). Local search strategies for satisfiability testing. DIMACS Series in Discrete Mathematics and Theoretical Computer Science, 26, 521-532.

21. Soh, T., Inoue, K., Banbara, M., \& Tamura, N. (2005). Experimental results for solving job-shop scheduling problems with multiple SAT solvers. In Proceedings of the 1st international workshop on distributed and speculative constraint processing (DSCP'05).

22. Taillard, E. D. (1993). Benchmarks for basic scheduling problems. European Journal of Operational Research, 64, 278-285.

23. Tamura, N., Taga, A., Kitagawa, S., \& Banbara, M. (2006). Compiling finite linear CSP into SAT. In Proceedings of the 12th international conference on principles and practice of constraint programming (CP 2006) (pp. 590-603).

24. Walsh, T. (2000). SAT v CSP. In Proceedings of the 6th international conference on principles and practice of constraint programming (CP 2000) (pp. 441-456). 